\title{
SOLUTIONS FOR EXTERIOR ORIENTATION IN PHOTOGRAMMETRY, A REVIEW
}

\author{
P. GRUSSENMEYER (pierre.grussenmeyer@ensais.u-strasbg.fr) \\ O. AL KHALIL (alkhalil@ensais2.u-strasbg.fr) \\ ENSAIS, Photogrammetry and Geomatics Group, Strasbourg, France
}

\begin{abstract}
The determination of the attitude, the position and the intrinsic geometric characteristics of the camera is known as the fundamental photogrammetric problem. It can be summarised in the determination of camera interior and exterior orientation parameters, as well as the determination of $3 D$ coordinates of object points. The term "exterior orientation" of an image refers to its position and orientation related to an exterior coordinate system. Several methods can be applied to determine the parameters of the orientation of one, two or more photos. The orientation can be processed in steps (as relative and absolute orientation) but simultaneous methods (as bundle adjustments) are now available in a majority of software packages. Several methods have also been developed for the orientation of single images. They are based in general on geometric and topologic characteristics of imaged objects.

In this paper we present a survey of classical and modern methods for the determination of the exterior parameters in photogrammetry, available for some of them as software packages with practical examples on the Internet. The presented methods are classified in three principal groups. In the first one, a selection of approximate methods for applications that do not require grand accuracy are presented. They are also used to calculate values required for iterative methods. In the second group, standard point-based methods derived from collinearity, coplanarity or coangularity conditions are shortly reviewed with an extend to line-based approaches. The third group represents orientation methods based on constraints and projective geometry concepts, which are more and more of interest for photogrammetrists. In the last section, the paper gives a summary of existing strategies for automatic exterior orientation in aerial photogrammetry.
\end{abstract}

KEYWORDS: exterior orientation, approximate solutions, photogrammetric conditions, projective geometry.

\section{General Presentation Of The Photogrammetric Problem}

The fundamental photogrammetric problem amounts to the determination of the interior and exterior orientation parameters of the camera and to the coordinates of object space points measured on photos $\left(\mathrm{M}^{\mathrm{c}}\right.$ Glone, 1989). For the interior orientation, two sets of parameters have to be considered. The first one contains the geometric parameters of the camera: the principal distance and the coordinates of the principal point. The second set includes the parameters that describe the systematic errors (as distortions or film deformations). The exterior orientation aims to define the position and rotation of the camera at the instant of exposure.

Several methods can be applied to determine the parameters of the orientation of one, two or more photos. The orientation can be processed in steps (relative and absolute orientation) but simultaneous methods (as bundle adjustments) are now available in a majority of software packages. For systems based on stereoscopic measurements, the stereomodel derives from a relative orientation, which is the equivalent to the clearing of vertical parallax on a stereoplotter. In this step, points coordinates are determined in an arbitrary coordinate

Grussenmeyer, P. \& Al Khalil, O. Solutions for exterior orientation in photogrammetry, a review.

The photogrammetric record, an international journal of photogrammetry. Accepted for publication, May 2002 
system of the stereomodel. The determination of points in the object coordinate system is done in a second step, known as absolute orientation, by applying a three-dimensional similarity transformation.

If we consider the orientation of a single image, the topological and geometrical characteristics of the imaged scene are used with the measurements in the image to determine the orientation parameters. These characteristics are considered as scene constraints (e.g. perpendicularity, parallelism, symmetry or co-planarity). The relationship between camera and object spaces is given by the perspective projection model of the camera (also known as the pinhole model). In fact, if homogenous coordinates are used, this relationship is a linear one. Points at infinity (or vanishing points) play a very important role in the computing of the projection matrix. Non calibrated images can also be used in this case.

In photogrammetry, three fundamental conditions are frequently used to compute the exterior orientation parameters. These conditions are known as collinearity, coplanarity and coangularity conditions. All the solutions based on the conditions mentioned so far use point coordinates as input data (this is valid even for linear solutions based on photogrammetric line extraction). But in many cases, the available control information has another form given as scene and/or camera constraints. The use of this type of control information characterises in general the applications of non-topographic photogrammetry where camera geometric constraints, object-space geometric and topologic constraints are used as input data. Most of the new proposed solutions use the constraints to compute the exterior orientation parameters from which robust algorithms for single image, couple and bloc images are derived. Some of these algorithms are discussed in this paper.

The problem of the determination of the exterior orientation parameters in computer vision is known as pose estimation problem. Research in this field aims in direct solutions to the problem of pose estimation by using a minimum amount of object information. Direct linear solutions for the pose estimation based on concepts of algebraic projective geometry are largely used. However, using projective geometry is not new and homogenous coordinates, one of its most important concepts, are used for deriving camera parameters as the equivalent to the Direct Linear Transformation equations.

GPS techniques can also be used as a possible solution of the exterior orientation problem, but only in aerial photogrammetry. Using GPS allows direct transformation of points into the mapping coordinate system. The main advantage of this method is the limitation of the iterative computation traditionally used to determine exterior orientation parameters. As a result, approximate values of exterior orientation parameters are not needed and the number of control points needed to compute these values is considerably reduced.

We consider in this paper both approximate and rigorous methods for determining exterior orientation parameters in aerial and terrestrial photogrammetry. As approximate solutions, we have included the Direct Linear Transformation (frequently used in photogrammetry and remote sensing), the Church method of space resection (used for single image resection), a simplified absolute orientation (used when no control points are available), a method of 3D conformal transformation (where a special parameterisation of the rotation matrix is proposed) and an approximate solution of spatial transformation (suitable when using incomplete control points). As rigorous adjustment methods, the three fundamental conditions in photogrammetry (collinearity, coplanarity and coangularity) are recapitulated. In the same section, based-point methods and line-based ones are discussed. Other solutions based on constraints and concepts of algebraic projective geometry are also tackled. In the last section, we give a general look of existing strategies used to carry out an automatic exterior orientation in aerial photogrammetry.

The various approaches are not independent. The relations between them as well as an overview of the different solutions presented in this paper are given in Fig. 1. A large set of references of papers and webpages is listed at the end of the paper to help the reader to go thoroughly into the problem of exterior orientation in photogrammetry. 


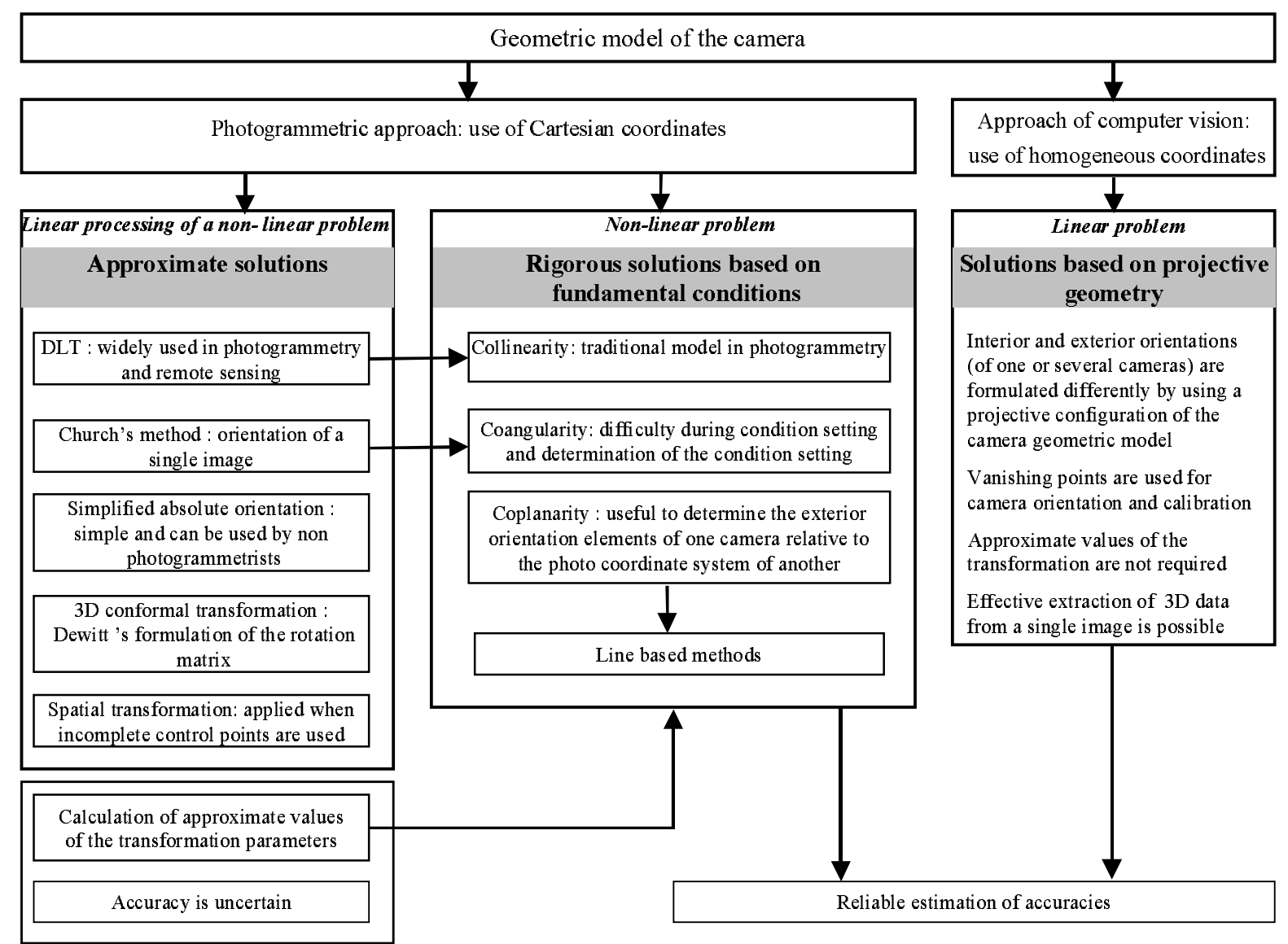

FIG. 1. Relations between different solutions for the exterior orientation in photogrammetry

\section{APPROXIMATE SOLUTIONS}

Approximate solutions are effective in applications that do not require rigorous design and estimation of data, as working with non-metric cameras for example. All these solutions are a linear processing of an originally non-linear problem (for example the relationship between image and object coordinates is considered as linear). Neither a calibrated camera nor initial approximations for the parameters are required. Such solutions are often used to compute approximate values of the exterior orientation parameters required for further rigorous adjustments. As examples of these solutions, we discuss about the following methods:

a) The Direct Linear Transformation (DLT), which is a method frequently used in photogrammetry and remote sensing,

b) The Church method proposed as a solution for single image resection (Slama, 1980),

c) A simplified absolute orientation method based on object-distances and vertical lines used when no control points are available. This method is largely applied in archaeology and architecture by nonphotogrammetrists due to its simplicity,

d) A method of 3D conformal coordinate transformations (Dewitt, 1996) where a special formulation of the rotation matrix as a function of the azimuth and tilt is proposed,

e) An approximate solution of the spatial transformation (Kraus, 1997) which is particularly suitable when incomplete control points are used.

\section{Direct Linear Transformation}

Originally proposed by Abdel-Aziz and Karara (1971), the Direct Linear Transformation (DLT) can be solved without supplying initial approximations for the transformation parameters, and is very suitable for projects based on non-metric cameras. The mathematical model of the DLT, derived from the collinearity equation (see the collinearity section), is a direct linear relationship between comparator coordinates and object coordinates. This model is based on the two following collinearity equations: 


$$
\begin{aligned}
& x^{\prime}-\delta_{x}=\frac{L_{1} X+L_{2} Y+L_{3} Z+L_{4}}{L_{9} X+L_{10} Y+L_{11} Z+1} \\
& y^{\prime}-\delta_{y}=\frac{L_{5} X+L_{6} Y+L_{7} Z+L_{8}}{L_{9} X+L_{10} Y+L_{11} Z+1}
\end{aligned}
$$

Where $x^{\prime}$ and $y^{\prime}$ are the image coordinates, and $(X, Y, Z)$ an arbitrary coordinate system in the object. $\delta_{x}$ and $\delta_{y}$ are the systematic errors (mainly lens distortions) in the image coordinate system. These equations can be considered as observation equations with 11 unknown parameters. They can be solved by an iterative method if more than six non-coplanar control points are available.

\section{The Church method of space resection}

This method (Slama, 1980) is one of the several derived approaches of single photo resection and requires three control points. The solution assumes that no geometric distortion exists at the three corresponding image points. Originally, this model has been derived from fundamental geometric properties of aerial photography, but it can also be used in close range applications. In this model, the angle between two given points seen from the perspective centre in the object space is equal to the angle between the images of these points, defined from the perspective centre in the photograph. In Fig. 2, this coangularity condition states that:

$$
\begin{aligned}
& A \hat{O B}=a \hat{o} b \\
& B \hat{O C}=b \hat{o} c \\
& A \hat{O C}=a \hat{o} c
\end{aligned}
$$

The parameters of the interior orientation are supposed to be known. The geometry of the pyramid is defined by the three points in the image or the object and the perspective centre. The solution of Church requires initial values of the exterior orientation parameters and follows a successive iteration procedure until the two pyramids coincide, i.e. until the equations (2) are satisfied.

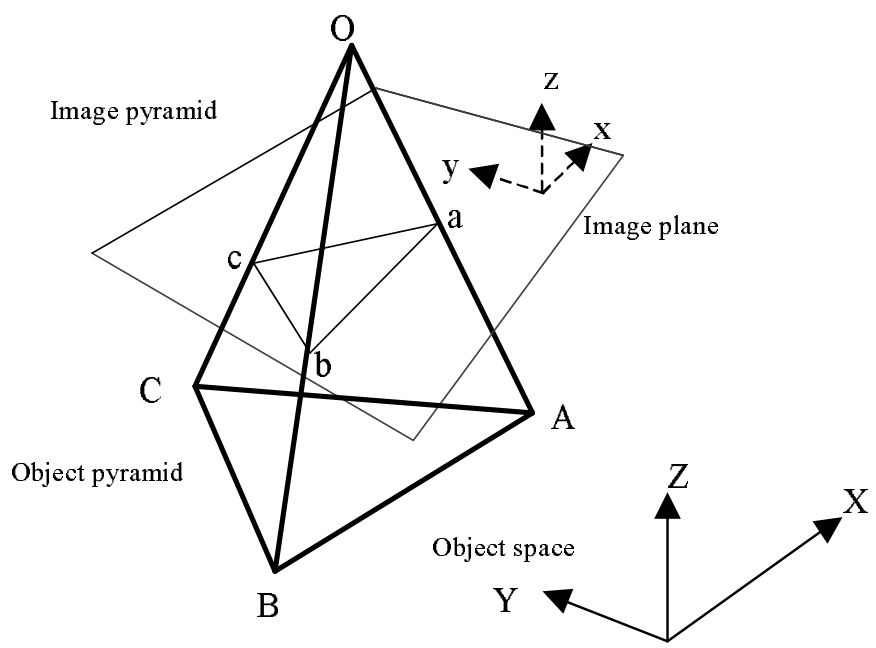

FIG. 2. Space resection

The algorithm of Church can take into account image distortions but in this case four or more control points are needed to apply the least squares method.

\section{Simplified absolute orientation}

When we apply a non-simultaneous method, a step of relative orientation is required at first, and the stereomodel is generated in an arbitrary coordinate system. The simplified absolute orientation does not require any control point to transform the model coordinate system into the object one. The algorithm consists in carrying out rotations and scaling of the stereomodel. In fact, two rotations have to be applied in order to orient the stereo model with the vertical line in the object space. To scale the stereomodel, a rotation and a given distance in the photo and the object space are required. This method is available in the TIPHON software package developed in 
Strasbourg, freely downloadable at http://photogeo.u-strasbg.fr/tiphon and in the web based ARPENTEUR software package available at www. arpenteur.net.

\section{$3 D$ conformal transformation}

The absolute orientation of a stereomodel is given by a 3D conformal transformation of points following a relative orientation. The seven unknown parameters of this transformation are the scale factor, the three spatial rotations represented by the rotation matrix of the photogrammetric model into the object coordinates system and translation of the model coordinate system into the object coordinate system.

The equations of the 3D conformal transformation are not linear, and a least squares iterative solution has to be applied. We then require initial values of the unknown parameters.

The calculation is done in three steps (Dewitt, 1996):

a) The scale factor is directly estimated from a ratio of distances :

$$
s=\frac{\text { distancein thereferencecoordinatesystem }}{\text { distancein the stereomodel coordinatesystem }}
$$

b) Rotation angles are determined in seven steps. The basic idea is the formulation of the rotation matrix as a function of the azimuth, tilt and swing. That requires a particular configuration of the distribution of the used control points to avoid the use of three collinear points. The orientation of the (XY) plane is evaluated at first from the azimuth and the tilt of both coordinate systems. The difference between the azimuths of these systems is used to find the swing. The final rotation matrix is determined by combining tilts, azimuths and swing resulting from the last step.

c) Translations are computed from common points between both coordinate systems.

Note: A computer program based on this method and given by Wolf and Dewitt (2000) is available at the following address: http://www.surv.ufl.edu/wolfdewitt/download.html.

\section{Approximate solution for spatial transformation}

If at least four homologue points in both image and object coordinate systems are available, the 12 parameters of the spatial transformation (nine dependent elements of a rotation matrix and three parameters of a translation) which maps image points into object ones, can be computed using the following linear transformation given in Kraus (1997):

$$
\mathbf{X}=\left(\begin{array}{c}
X_{0} \\
Y_{0} \\
Z_{0}
\end{array}\right)+\left(\begin{array}{lll}
a_{11} & a_{12} & a_{13} \\
a_{21} & a_{22} & a_{23} \\
a_{31} & a_{32} & a_{33}
\end{array}\right) \mathbf{x}^{\prime} \Rightarrow \mathbf{X}=\mathbf{X}_{\mathbf{0}}+\mathbf{A x}^{\prime}
$$

From this relationship we can notice that four non-coplanar points are required to determine the unknown parameters.

The matrix $\mathbf{A}$ represents the rotation matrix multiplied by a scale factor. This factor can be computed using the following equation:

$$
m=\frac{\sqrt{a_{11}^{2}+a_{12}^{2}+a_{13}^{2}+\ldots \ldots+a_{33}^{2}}}{3}
$$

Note: A JAVA ${ }^{\mathrm{TM}}$ version of this method can be downloaded at http://photogeo.ustrasbg.fr/photogrammetry. In this program, Cartesian and homogenous coordinates are used to compute the 12 unknown parameters of the transformation. 


\section{FUNDAMENTAL PHOTOGRAMMETRIC CONDITIONS}

\section{Collinearity}

The collinearity condition expresses the basic relationship in which an object point and its image lies on a straight line passing through the perspective centre:

$$
\left(\begin{array}{c}
x^{\prime}-x_{0}^{\prime} \\
y^{\prime}-y_{0}^{\prime} \\
-c
\end{array}\right)=k \mathbf{R}\left(\begin{array}{c}
X-X_{C} \\
Y-Y_{C} \\
Z-Z_{C}
\end{array}\right) \text { or } \mathbf{a}^{\prime}=k \mathbf{R} \mathbf{a}
$$

- $\mathbf{a}$ is the vector from the perspective centre to the point expressed in the object space coordinate system,

- $X, Y, Z$ are the coordinates of the object-point and $X_{C}, Y_{C}, Z_{C}$ are the coordinates of the perspective centre,

- $\quad \mathbf{a}^{\prime}$ is the corresponding vector expressed in the camera space coordinate system ( $c$ is the principal distance of the camera, $x_{0}^{\prime}$ and $y_{0}^{\prime}$ are the coordinates of the principal point),

- $\quad \mathbf{R}$ is the rotation matrix and $k$ is the scale factor.

This collinearity equation contains the coordinates of the object point as well as the exterior orientation and the interior orientation parameters. Image coordinates of each point are considered as observations. All unknown parameters of a project can be grouped in a simultaneous solution (with given or unknown interior orientation elements).

The functional model of standard aerial block adjustments is a typical application of the collinearity equation

\section{Coplanarity}

In most photogrammetric problems, object points are recorded on two or more photographs. For two photos, the two conjugate rays defined on each object point must be coplanar. The corresponding mathematical condition, known as the coplanarity equation, implies that the two camera stations, the two image points, and the object point are in a same epipolar plane. The coordinates of the object point do not appear in the equation, so no approximations for the coordinates are needed.

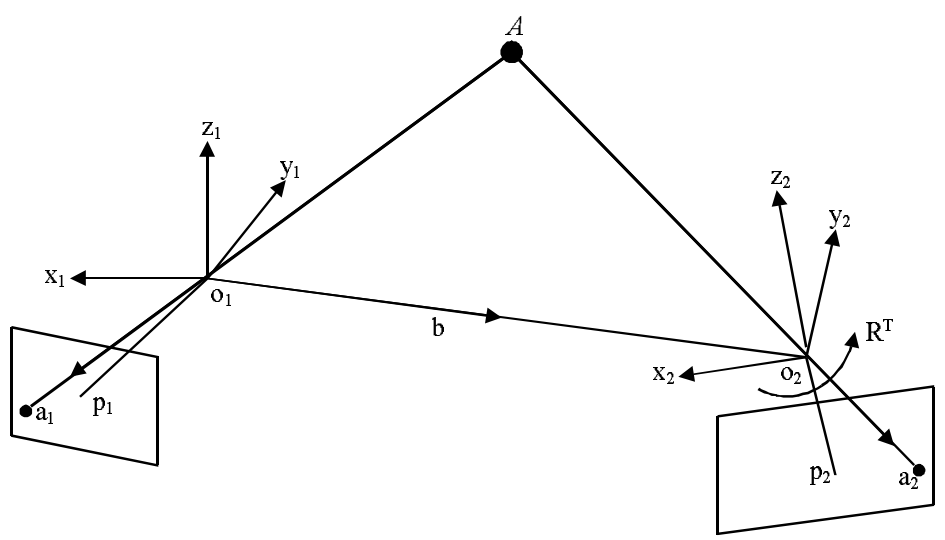

FIG. 3. Coplanarity

From Fig. 3, the equations are written as a function of the base vector (the vector $\mathbf{b}$ between the two perspective centres) and each image point vector $\left(\mathbf{a}_{1}\right.$ and $\mathbf{a}_{2}$ ). These vectors, which are coplanar, are given in the photo coordinate system of the left image whose projection centre is $\mathrm{O}_{1}$ :

$$
\begin{aligned}
& \mathbf{a}_{1}=-\lambda\left(\begin{array}{lll}
\mathrm{x}_{1}^{\prime} & \mathrm{y}_{1}^{\prime} & -\mathrm{c}
\end{array}\right)^{\mathrm{T}} \\
& \mathbf{a}_{2}=-\mu \mathbf{R}^{\mathrm{T}}\left(\begin{array}{llll}
\mathrm{x}_{2}^{\prime} & \mathrm{y}_{2}^{\prime} & -\mathrm{c}
\end{array}\right)^{\mathrm{T}} \quad \mathbf{b}=\left(\begin{array}{llll}
b_{x} & b_{y} & b_{z}
\end{array}\right)^{\mathrm{T}}
\end{aligned}
$$

where $\mathbf{R}$ is the rotation matrix. $\lambda$ and $\mu$ are scalars greater than zero whose values influence the parallax vector (Cooper and Robson, 1996). 
The volume of the parallelepiped formed by these three vectors must be equal to zero:

$$
\mathbf{b} \cdot\left(\mathbf{a}_{1} \times \mathbf{a}_{2}\right)=\mathbf{0}
$$

These equations contain 12 unknowns: three coordinates and three orientation angles for each of photo. The coplanarity equation is useful to determine the exterior orientation elements of a camera relative to the photo coordinate system of another one. We will see later that most of the line-based methods are built on the coplanarity condition.

\section{Coangularity}

We have seen in the method of Church an application of the coangularity condition, suitable for single image application. An easy, fast and rigorous algorithm for finding and computing the essential independent conditional equations that link object and image spaces has been developed in (Wang, 1992). The functional model of coangularity can be derived from Fig. 4:

$$
F(\mathbf{U}, \mathbf{u})=\phi_{A B}-\varphi_{a b}=0
$$

where $\phi_{A B}$ is the angle between the projection centre and the two object points $A$ and $B . \varphi_{a b}$ is the corresponding angle in image space between the projection centre and the two corresponding image points $a$ and $b$.

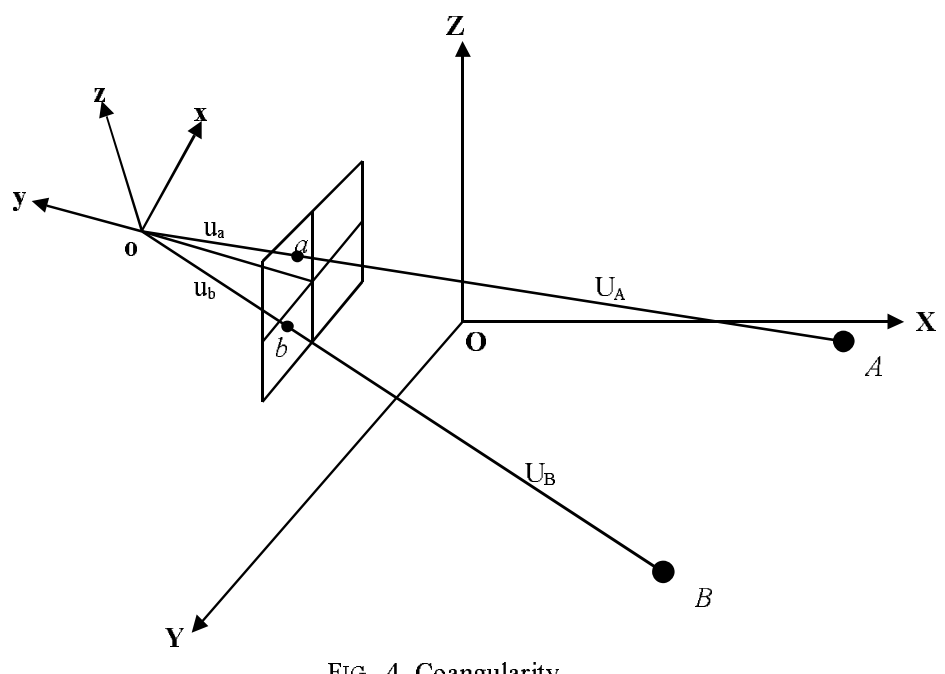

FIG. 4. Coangularity

In his approach, the coangularity condition combined with a transformation technique of conditional equations into observation equations is developed. Applying this method on a block adjustment allows a block of high quality (compared with other conditions in photogrammetry) and a fast determination of the coordinates of ground points. Since rotations are not used as parameters in the functional model, their approximate values are not needed. However, the transformation of the conditional equations into observations ones is not easy and a problem of ambiguity in angle values can appear because the author uses the cosinus functions to construct the basic adjustment functional model.

\section{Line-based methods}

Within the context of automation in digital photogrammetry, it is easier to extract linear features than apply point-based methods. Linear features are the entities the most common in the man-made environment. Particular conditions such as parallelism, coplanarity, orthogonality, horizontal or vertical situations makes them usable as control information to compute the parameters of the exterior orientation. In this case, no point correspondence is required. Most of the line-based methods are indirectly based on the coplanarity condition.

a) Straight line representation in photogrammetry

Theoretically, a straight line has six degrees of freedom in the 3D Euclidean space: the coordinates of an arbitrary point lying on this line and the components of its orientation vector. In fact, this representation suffers from an over-parameterisation. For that reason, two constraint equations are imposed: the arbitrary point is often chosen as the nearest point to the origin in the object space coordinate system. The orientation 
vector of the line is constrained to be a unit vector. Let $\mathbf{c}=\left(\begin{array}{llll}c_{X} & c_{Y} & c_{Z}\end{array}\right)^{T}$ be the arbitrary point and $\boldsymbol{\beta}=\left(\begin{array}{llll}\beta_{X} & \beta_{Y} & \beta_{Z}\end{array}\right)^{T}$ be the orientation vector, then the constraint equations are given as:

$$
\begin{aligned}
& |\boldsymbol{\beta}|=\beta_{X}^{2}+\beta_{Y}^{2}+\beta_{Z}^{2}=1 \\
& \boldsymbol{\beta}^{\mathrm{T}} \cdot \mathbf{c}=\beta_{X} c_{X}+\beta_{Y} c_{Y}+\beta_{Z} c_{Z}=0
\end{aligned}
$$

However, the disadvantage of this representation is that the sign of the orientation vector is undetermined. In the camera space coordinate system, the image coordinates of its endpoints and the principal distance of the camera define the straight line. Another representation has been proposed in (Van Den Heuvel, 1997) where the image coordinates of end points are replaced by the normal vector to the interpretation plane of the line.

b) Exterior orientation parameters in line-based methods

In this section, two different line-based approaches are presented. The first one is based on constrained linear features extraction, while the second one exploits the available assumption about the object geometry to determine the parameters of the exterior orientation.

- Constrained linear features extraction

A new algorithm has been proposed in Chen and Shibasaki (1998) to determine the parameters of the exterior orientation of a camera whose interior orientation and distortion parameters are known. Their mathematical model is based on the coplanarity condition and geometric constraints and computes the parameters of the exterior orientation in two steps. After determining the attitude of the camera, its position is obtained by using at least three control lines (the term "control lines" is used for lines constrained to be horizontal or vertical lines). The basis of the proposed functional model is the coplanarity condition, where the perspective centre, the line in object space and its corresponding image form a plane. The normal to this plane in both object and image space, with coplanarity condition, are used to establish the mathematical model. The normal is then constrained and written as a function of the exterior orientation parameters. Additional geometric constraints, such as horizontality and verticality of lines, are imposed on the control lines to compute the parameters of interest. However, two control points per line are required when applying this model. This is the principal disadvantage of the method because a big number of such points is required to increase the accuracy. In fact, the principal goal of this model is to show the efficiency of digital photogrammetry by linear features automatic extraction.

- Volumetric approach

A method based on the extraction of two sets of parallel lines in the object space has been proposed in Van Den Heuvel (1997). The lines are coplanar and form a parallelogram. The determination of the parameters of the exterior orientation is done in two separated steps. The algorithm developed by Abidi and Chandra (1995) in a project which aims "a new efficient and direct solution for pose estimation using quadrangular targets" is the basis of the proposed functional model of this method. As in the nonsimultaneous method, a relative orientation step based on a volumetric approach is required. In fact, this method is suitable for applications where polyhedral objects with parallel edges are dominant. First of all, in the relative orientation step, the ratios of the distances from the perspective centre to the corners of the parallelogram are computed. This calculation is based on a volumetric approach because it requires the calculation of the volumes of the tetrahedrons formed of the three corners of the parallelogram and the projection centre. This step allows the creation of a model in an arbitrary coordinate system. In the second step (similar to the absolute orientation in the non-simultaneous method) we compute the position and orientation of the image in the object space by applying a threedimensional similarity transformation. It should be noted that interior orientation and camera calibration are assumed to be known. This approach can be used as a solution for 3D reconstruction of object from single images. 


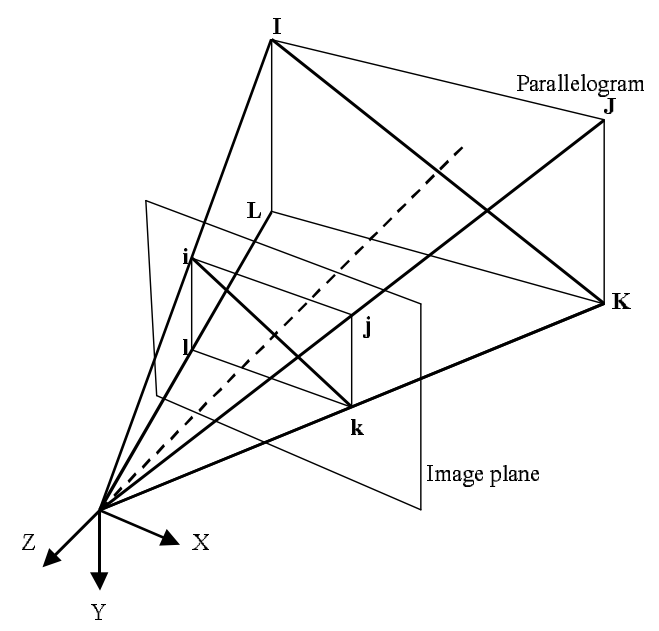

FIG. 5. Volumetric approach

\section{PROJECTIVE GEOMETRY CONCEPTS AS A SOLUTION OF THE EXTERIOR ORIENTATION PROBLEM}

Considering perspective image projection, the geometry of imaged objects is distorted and the perspective transformation of geometric shapes cannot be described using traditional Euclidean geometric aspects. By the use of projective geometry, all the important phenomena of this transformation can be taken into account (Mundy and Zisserman, 1992). Some projective geometry-based solutions of the orientation of one, two and three images are given in Förstner (2000). The following section is focused on concepts of algebraic projective geometry.

\section{Homogenous coordinates}

In the Euclidean plane, a point $\left(A \in R^{2}\right)$ is represented by its Cartesian coordinates $\left(x^{\prime}, y^{\prime}\right)$. The same point is represented in the projective plane by the following triple of coordinates $(X, Y, W)$ called homogenous coordinates of $A$. They provide a convenient way to replace the non -linear formulation of the conic projection. Relations between the two types of coordinates can be written as:

$$
\begin{aligned}
& X=\frac{x^{\prime}}{W} \\
& Y=\frac{y^{\prime}}{W}
\end{aligned}
$$

As an equivalent to the $D L T$ solution, the homogenous coordinates were used for deriving camera parameters and to calibrate non-metric cameras (Strat, 1984). As another example, a direct model to determine the orientation parameters of a non-metric camera is proposed in Abdel-Latif and Elsonbaty (1996). This model uses homogenous coordinates of four coplanar points and leads to a simple direct solution of the orientation problem.

\section{Projection matrix}

A perspective projection camera (or pinhole camera which presents a central projection) is represented in homogeneous coordinates by a $(3 \times 4)$ matrix. This matrix describes the perspective projection from the Euclidean 3D space to an image. The transformation between the image and the object coordinate systems is represented by using the $(3 \times 4)$ projection matrix $\mathbf{P}$ : 


$$
\left(\begin{array}{c}
x^{\prime} \\
y^{\prime} \\
1
\end{array}\right)=\mathbf{C}\left(\begin{array}{cccc}
1 & 0 & 0 & 0 \\
0 & 1 & 0 & 0 \\
0 & 0 & 1 & 0
\end{array}\right)\left[\begin{array}{cc}
\mathbf{R} & \mathbf{T} \\
\mathbf{0}^{\mathbf{T}} & 1
\end{array}\right]\left(\begin{array}{c}
X_{w} \\
Y_{w} \\
Z_{w} \\
1
\end{array}\right)=\mathbf{C}\langle\mathbf{R} \mid \mathbf{T}\rangle\left(\begin{array}{c}
X_{w} \\
Y_{w} \\
Z_{w} \\
1
\end{array}\right)=\mathbf{P}\left(\begin{array}{c}
X_{w} \\
Y_{w} \\
Z_{w} \\
1
\end{array}\right)
$$

where $\mathbf{P}(3 \times 4)$ is the projection matrix, $\mathbf{C}(3 \times 3)$ is the camera calibration matrix (an upper triangular matrix), $\mathbf{R}(3 \times 3)$ is the rotation matrix which describes the orientation of the camera, and $\mathbf{T}(3 \times 1)$ represents the translation vector.

The matrix $\mathbf{P}$ has 11 degrees of freedom and consists of:

a) orientation and position parameters of the camera relative to the object coordinate system (six parameters of the exterior orientation: three Euclidean coordinates of the projection centre defined by $\mathbf{T}$ and the three angles of rotation presented by the rotation matrix $\mathbf{R}$ ), and

b) the matrix of camera calibration $\mathbf{C}$ (equation 13) which contains five parameters of the interior orientation (the principal distance $f$, the coordinates of the principal point $\left(x_{0}^{\prime}, y_{0}^{\prime}\right)$, a ratio $r$ between the vertical and horizontal scale factors which describe the scale difference of the $x$ ' and $y$ ' coordinates, and $k$ the image skew parameter.

$$
C=\left(\begin{array}{ccc}
f & k & x_{0}^{\prime} \\
0 & r f & y_{0}^{\prime} \\
0 & 0 & 1
\end{array}\right)
$$

\section{Planar homography}

Describing a plan-to-plan projection (also known as a plan projective transformation) is a particular case of the general central projection, interesting in particular when planar surfaces are imaged. The (3x3) homography matrix can be computed using four points on the object plane together with their mapped points in the image.

Because it is not possible to extract 3D projective quantities from a single image if the imaged scene is not planar (at least locally), the homography plays a very important role in the extracting of 3D geometric data from in this case.

\section{Vanishing point and vanishing line: definition and detection}

By considering a perspective projection, the parallel straight lines in space converge to a point in the image plane named the vanishing point. Vanishing points are used to determine the direction of the corresponding set of parallel lines, feature correspondence and to compute the projection matrix. The union of vanishing points defines the horizon line, called the vanishing line (Fig. 6).

Vanishing point and the vanishing line are important aspects when a single image is used to recover the 3D model of the imaged scene.

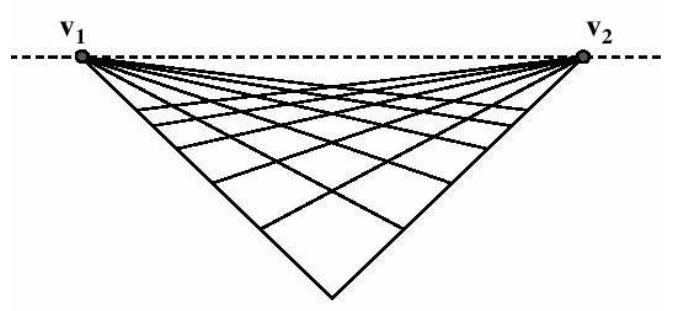

FIG. 6. Vanishing point and vanishing line 
Most of the vanishing point detection algorithms are based on the Gaussian sphere. This sphere is a unit sphere whose centre is the projection centre (pinhole camera model). The projection of a vanishing point onto this sphere defines a vector which has the same direction as the parallel lines in space. These algorithms can be applied to imagery with strong perspective effects and with little noise of texture, but they can fail in the contrary cases.

A method using geometric object information to detect the vanishing point has been proposed in Van Den Heuvel (1998). This method overcomes the disadvantages of Hough transformation-based methods. The perpendicularity assumption between three directions in the object space, which correspond to three vanishing points, is the key idea of this method. However, this technique is more computationally expensive compared to the existing ones.

In Leung and McLean (1996), a technique that differentiates mutual intersection of many lines in the image (corners of building for example) from true vanishing points is presented. The discriminating is based on the identification of invariant properties of vanishing points in multiple images of a scene. Relaxation labelling is used to match the vanishing points of two images of a scene.

\section{Using vanishing points to recover the camera calibration and the projection matrix}

We assume that images are obtained by perspective projection, and in addition that three vanishing points associated with three mutually perpendicular directions in the object space may be determined from the images. Regarding the camera calibration matrix, the skew parameter is assumed to be zero and the aspect ratio is considered as known. Under these assumptions, the 11 freedom degrees of the projection matrix are reduced to eight.

It is shown in Cipolla et al. (1999) and in Bauer and Voss (1999) that:

a) the three vanishing points corresponding to three perpendicular directions in the object space can be used to compute the camera calibration matrix. In fact, the orthocentre of the triangle formed by these vanishing points represents the principal point (the intersection of the heights of this triangle).

b) the use of this principal point ( $\mathrm{pp}$ in Fig. 7) with two vanishing points allows the computation of the focal length.

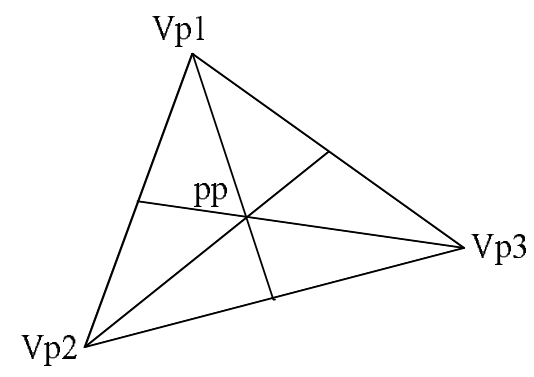

FIG. 7. The vanishing self-polar triangle

The relation between an object point $\mathbf{X}$ and its corresponding point in the image plane $\mathbf{x}$ is described by using the projection matrix $(\mathbf{P})$ :

$$
\begin{gathered}
\lambda_{i} \mathbf{x}=\mathbf{P X} \\
\lambda_{i}\left(\begin{array}{c}
x_{i}^{\prime} \\
y_{i}^{\prime} \\
1
\end{array}\right)=\left(\begin{array}{llll}
p_{11} & p_{12} & p_{13} & p_{14} \\
p_{21} & p_{22} & p_{23} & p_{24} \\
p_{31} & p_{32} & p_{33} & p_{34}
\end{array}\right)\left(\begin{array}{c}
X_{i} \\
Y_{i} \\
Z_{i} \\
1
\end{array}\right)
\end{gathered}
$$

where $\lambda_{i}$ is a scale factor.

If we assume that $\left(\mathbf{v}_{x}, \mathbf{v}_{y}, \mathbf{v}_{z}\right)$ are the vanishing points of the axes (X, Y, Z), we can notice that the three first columns of $\mathbf{P}$ represent these vanishing points (Criminisi et al., 1999). Coefficients of the fourth column of $\mathbf{P}$ depend on the position of the world coordinate system relative to the camera coordinate system (it represents the projection of the origin of the world coordinates system).

Grussenmeyer, P. \& Al Khalil, O. Solutions for exterior orientation in photogrammetry, a review. The photogrammetric record, an international journal of photogrammetry. Accepted for publication, May 2002 
In Criminisi (1999), the above approach is used to extract 3D geometric data from single views. To apply the proposed approach, the plane must be the dominant geometric entity in the scene: the plane projective transformation (homography) and vanishing points and lines are the basic elements. A new definition of the object space is given as a collection of three planes in three different directions. This description allows a complete 3D construction of the imaged scene. The projection matrix is written as function of the three vanishing points associated with three axes of a local coordinate system and with three scale factors:

$$
\mathbf{P}=\left(\begin{array}{llll}
\alpha_{x} \mathbf{v}_{x} & \alpha_{y} \mathbf{v}_{y} & \alpha_{z} \mathbf{v}_{z} & \mathbf{0}
\end{array}\right)
$$

$\left(\mathbf{v}_{x}, \mathbf{v}_{y}, \mathbf{v}_{z}\right)$ are the vanishing points and $\left(\alpha_{x}, \alpha_{y}, \alpha_{z}\right)$ are the scale factors.

o represents the origin of the image coordinate system. This point must not lie on any of the vanishing lines determined by the vanishing points $\left(\mathbf{v}_{x}, \mathbf{v}_{y}, \mathbf{v}_{z}\right)$. It is convenient to choose:

$$
\mathbf{0}=\frac{\mathbf{v}_{x} \times \mathbf{v}_{y}}{\left|\mathbf{v}_{x} \times \mathbf{v}_{y}\right|}
$$

The computation of scale factors $\left(\alpha_{x}, \alpha_{y}, \alpha_{z}\right)$ is done by using five reference points of which four points lie in a reference plane and one lies out of this plane (a reference height can also be used). It is important to notice that the first, second and fourth columns of the projection matrix define the homography that relies the reference plane in the image and object space. When there are no reference points, this decomposition of the projection matrix is useful to compute the two first scale factors by using a square form in the object with a known size.

\section{AUTOMATIC EXTERIOR ORIENTATION}

In aerial photogrammetry, the combination of aerial photographs with existing information from cadastral or GIS databases is more and more used to automate the exterior orientation of these photographs (Fig. 8).

In Smith and Park (2000), a technique based on the extraction and automatic matching of linear features is proposed to determine the absolute and exterior orientation parameters of aerial photographs. This technique is interesting because linear entities are dominant features in aerial photographs (as roads for example) and they can be extracted automatically from digital photos. The linear entities, given in a 3D geometric reference frame, replace the traditional control points. The matching of the homologous linear feature is done automatically by the application of a gross transformation followed by an iterative adjustment whose aim is to obtain the best matching. The parameters of the absolute orientation are then calculated by using a 3D transformation. The computation of the exterior orientation parameters are based on a perspective transformation.

Automatic exterior orientation can be carried out by combination of aerial photographs and existing topographic databases (Höhle and Potuckova, 2001). Control data are the roads crossing points. The (X, Y) coordinates of these points are automatically extracted from a topographic map. With regard to the $(\mathrm{Z})$ coordinates, they are extracted from the height information recorded in the topographic database. The previous stage is followed by an automatic extraction and matching of points in both orthoimages and aerial photographs. The last step consists in applying a spatial resection, which aims to compute the exterior orientation parameters using the matched points.

Image matching between aerial images and corresponding orthoimages both recorded in a GIS database is the the basic idea of the approach proposed in (Shan, 2000). Elevation information is extracted from a Digital Terrain Model. By applying a robust bundle adjustment, the matched points are used to compute the exterior orientation parameters.

In (Drewniok and Rohr, 1996), manhole covers are extracted automatically from the images, and used to carry out the automatic exterior orientation of aerial photographs. 3D object coordinates of landmarks are automatically extracted from a cadastral database. Manhole covers are circular landmarks, which can be found abundantly in urban environments, and they can be extracted and matched with precision in aerial photographs.

In (Läbe and Ellenbeck, 1996), the control points used for the automatic exterior orientation are 3D edges of 3D wireframe models of buildings or group of buildings. Each 3D edge is described by means of 3D points recorded in a database. The proposed algorithm consists in the automatic extraction of control points in the 
image followed by a matching stage and a spatial resection, which aims to compute the exterior orientation parameters. 


\begin{tabular}{|c|c|}
\hline Basic data & Automatic exterior orientation \\
\hline \multirow[t]{3}{*}{$\begin{array}{l}\text { and } \\
\text { Cadastral or GIS databases : } \\
-\quad \text { orthoimages } \\
-\quad \text { vector maps } \\
-\quad \text { DTM or height information }\end{array}$} & $\begin{array}{l}\text { Stage 1 } \\
\text { Automatic extraction of patches in aerial photographs } \\
\text { Automatic extraction of patches in orthoimages } \\
\text { Patches : } \\
\text { - } \quad \text { 3D edges of buildings wireframe models } \\
\text { - } \quad \text { crossroads } \\
\text { - linear or curvilinear features } \\
\text { - } \quad \text { manhole covers }\end{array}$ \\
\hline & $\begin{array}{l}\text { Stage } 2 \\
\text { a) Patches matching } \rightarrow \text { Image coordinates } \\
\text { b) 3D coordinates setting of matched patches: } \\
\text { - from orthoimages and elevation data } \\
\text { or } \\
\text { - } \quad \text { 3D GPS patches measured in the field } \\
\text { c) Image coordinates } \rightarrow \text { Object coordinates }\end{array}$ \\
\hline & $\begin{array}{ll}\text { Stage } 3 \\
\text { Bundle adjustment } \rightarrow \text { Exterior orientation parameters }\end{array}$ \\
\hline
\end{tabular}

FIG. 8. Overview of approaches for automatic exterior orientation.

\section{CONCLUSION}

In this paper, classical and modern methods used to determine the camera exterior orientation parameters have been presented and classified in three principal groups. Classification criteria depend on control geometric entity types and on the mathematical basis used to describe the relation between the image and the object. Approximate solutions have been tackled. These solutions can stand alone in particular cases (when a grand accuracy is not required) but they are usually prefered as an introduction to rigorous approaches.

The fundamental photogrammetric conditions (collinearity, coplanarity and coangularity) have been reviewed. More recent solutions, such as line-based and projective geometry-based methods have completed the overview by detailing aspects as homogeneous coordinates, vanishing points, projection matrix, etc.

We can notice the actual tendency in photogrammetry towards single view modelling. Given that the determination of camera intrinsic and exterior parameters constitute the first step of the modelling procedure, the solutions based on projective geometry aspects can be very useful in this context.

Other solutions using ground control features from existing databases as orthoimages, DTM interpretation operations or $3 \mathrm{D}$ wireframe models of buildings can be investigated to automate the determination of the parameters of the exterior orientation.

\section{REFERENCES}

ABDEL AZIZ, Y. I. and KARARA, H. M., 1971. Direct linear transformation from comparator coordinates into object-space coordinates. Close-range photogrammetry. American Society of Photogrammetry, Falls Church, Virginia, 433 pages: 1-18.

ABDEL-LATIF, M. E. and ELSONBATY, A., 1996. Using homogeneous coordinates to solve the problems of determining the orientation parameters of non-metric cameras and the reconstruction of space models. International Archives of Photogrammetry and Remote Sensing, 31(3): 1-7.

ABIDI, M. A. and CHANDRA, T., 1995. A new efficient and direct solution for pose estimation using quadrangular targets: algorithm and evaluation. http://www.computer.org/tpami/tp1995/i0534abs.htm [Accessed: 10 Sept. 2001].

BRAUER, C. and VOSS, K., 1999. Digital image processing group. Publications: http://ipc08.inf.uni-jena.de/pframe.phtml/e/public.html [Accessed: 10 Sept. 2001].

CHEN, T. and SHIBASAKI, R. S., 1998. Determination of camera's orientation parameters based on line features. International Archives of Photogrammetry and Remote Sensing, 32(5): 23-28.

CIPOLLA, R., ROBERTSIN, D., BOYER, E. 1999. Roberto Cipolla, publications. http://svr-www.eng.cam.ac.uk/ cipolla/publicats.html [Accessed: 10 Sept. 2001]

COOPER, M. A. R., ROBSON, S., 1996. Theory of close range photogrammetry. Chapter 2 in Close range photogrammetry and Machine vision (Ed. K. B. Atkinson). University College London. Whittles Publishing. 371 pages: 9-51.

Grussenmeyer, P. \& Al Khalil, O. Solutions for exterior orientation in photogrammetry, a review.

The photogrammetric record, an international journal of photogrammetry. Accepted for publication, May 2002 
CRIMINISI, A., 1999. Visual Geometry Group. Publications. http://imogen.robots.ox.ac.uk:20000/ geoff/papers.cgi [Accessed : 10 Sept. 2001]

CRIMINISI, A., REID, I., ZISSERMAN, A., 1999. Visual Geometry Group, publications. http://imogen.robots.ox.ac.uk 20000/ geoff/papers.cgi [Accessed : 10 Sept. 2001]

DEWITT, B. A., 1996. Initial approximations for the three-dimensional conformal coordinate transformation. Photogrammetric Engineering \& Remote Sensing, 62(1): 79-83.

DREWNIOK, C., ROHR, K., 1996. Automatic exterior orientation of aerial images in urban environments. International Archives of Photogrammetry and Remote Sensing, 31(3): 146-152.

FÖRSTNER, W., 2000. New orientation procedures. International Archives of Photogrammetry and Remote Sensing, 33(B3): 297-304.

VAN DEN HEUVEL, F. A., 1997. Exterior orientation of a single image based on line measurements of a rectangle. http://www. geo.tudelft.nl/frs/architec/rectangle [Accessed : 10 Sept. 2001]

VAN DEN HEUVEL, F. A., 1998. Vanishing point detection for architectural photogrammetry. International Archives of Photogrammetry and Remote Sensing, 32(5): 652-659.

HÖHLE, J., POTUCKOVA, M., 2001. Towards full automatic production of orthoimages. Photogrammetrie, Fernerkundung, Geoiformation, 6 : 397-404.

KRAUS, K., 1997. Procedures for point measurement in small areas. Photogrammetry. Volume 2. Advanced Methods and Applications. Fourth edition. Dümmler Verlag, Bonn. 466 pages: 43-112.

LÄBE, T., ELLENBECK, K. H., 1996. 3D-wireframe models as ground control points for the automatic exterior orientation. http://www.ipb.uni-bonn.de/ipb/lit/abstracts96/laebe96.database for amor.html [Accessed: 14 Jan. 2002]

LEUNG, J., MCLEAN, G., 1996. Vanishing point matching. http://persweb.direct.ca/jleung/icip96/icip96.html [Accessed: 10 Sept. 2001]

$\mathrm{M}^{\mathrm{C}} \mathrm{GLONE}$, J. C., 1989. Analytic data-reduction schemes in non-topographic photogrammetry. Chapter 4 in Non-topographic photogrammetry (Ed. American Society for Photogrammetry and Remote Sensing). Falls Church, Virginia. 445 pages: 37-55.

MUNDY, J. and ZISSERMAN, A., 1992. Course Notes. http://www.cs.cmu.edu/ ph/869/www/notes/notes.html [Accessed: 10 Sept. 2001]

SHAN, J., 2000. Automatic image orientation by using GIS data. International Archives of Photogrammetry and Remote Sensing, 33(3): 831836.

SLAMA, C. C. (Ed.), 1980. Manual of Photogrammetry, $4^{\text {th }}$ edition. American Society for Photogrammetry \& Remote Sensing, Falls Church, Virginia. 1056 pages.

SMITH, M. J. and PARK, D. W. G., 2000. Absolute and exterior orientation using linear features. International Archives of Photogrammetry and Remote Sensing, 33(B3): 850-857.

STRAT, T. M., 1984. Recovering the Camera Parameters from a Transformation Matrix. Image Understanding Workshops, 84: 264-271.

WANG, Y., 1992. A rigorous photogrammetric adjustment algorithm based on co-angularity condition. International Archives of Photogrammetry and Remote Sensing, 29(B5): 195-202.

WOLF, P. R. and DEWITT, B. A., 2000. Elements of photogrammetry, with applications in GIS. McGraw-Hill, New-York. 608 Pages.

\section{Résumé}

La détermination de l'attitude, de la position et des caractéristiques intrinsèques de la chambre photographique constitue un problème fondamental en photogrammétrie. Il se résume à la détermination des paramètres de l'orientation de la chambre de prise de vue (paramètres des orientations externe et interne), ainsi $q u$ 'à la détermination des coordonnées $3 D$ des points de l'objet. L'orientation externe se rapporte à la détermination de la position et de l'orientation d'une chambre par rapport à un système externe de coordonnées. Différentes méthodes peuvent être utilisées pour calculer les éléments d'orientation externe d'une photo, d'un couple ou de plusieurs photos. Le calcul de l'orientation peut être réalisé par étapes (par exemple les orientations relative et absolue) mais les méthodes simultanées (la compensation par faisceaux par exemple) sont actuellement proposées dans la plupart des logiciels. Plusieurs méthodes ont aussi été développées pour l'orientation d'images isolées. Elles sont basées en général sur les caractéristiques géométriques et topologiques des objets photographiés.

Dans cet article nous présentons un ensemble de méthodes classiques et modernes pour la détermination des paramètres de l'orientation externe, certaines d'entre elles étant téléchargeables sous la forme d'applications sur l'Internet. Les méthodes présentées sont classées en trois groupes principaux. Le premier groupe contient une sélection de méthodes approximatives utilisées d'habitude quand une grande précision n'est pas exigée, ou encore pour calculer des valeurs approchées des paramètres extrinsèques requises pour les méthodes itératives rigoureuses. Dans le deuxième groupe, nous rappelons brièvement les fondements des méthodes basées sur les conditions photogrammétriques fondamentales la colinéarité, la coplanéité et la coangularité). Dans ce groupe, les méthodes basées sur l'extraction des points ou des lignes, sont également abordées. Le troisième groupe traite des méthodes d'orientation basées sur les contraintes et les concepts de la géométrie projective, de plus en plus utilisées par les photogrammètres. Le dernier paragraphe se rapporte aux méthodes destinées à automatiser le calcul de l'orientation externe en photogrammétrie aérienne..

Grussenmeyer, P. \& Al Khalil, O. Solutions for exterior orientation in photogrammetry, a review.

The photogrammetric record, an international journal of photogrammetry. Accepted for publication, May 2002 